\title{
The NGC 2419 project: preliminary results on stellar variability
}

\author{
M. Di Criscienzo ${ }^{1}$, C. Greco ${ }^{2}$, M. Dall' Ora ${ }^{1}$, V. Ripepi ${ }^{1}$, \\ G. Clementini ${ }^{2}$, I. Musella ${ }^{1}$, M. Marconi ${ }^{1}$, L. Federici ${ }^{2}$, \\ L. Di Fabrizio ${ }^{2}$, Baldacci $^{2}$ and M. Maio ${ }^{2}$ \\ ${ }^{1}$ INAF-Osservatorio Astronomico di Capodimonte, Napoli, Italy \\ email: dicrisci@na.astro.it \\ ${ }^{2}$ INAF-Osservatorio Astronomico di Bologna, Italy \\ ${ }^{3}$ INAF- Telescopio Nazionale Galileo, Santa Cruz de La Palma, Spain
}

\begin{abstract}
We have combined $B, V, I$ time-series photometry from three different telescopes (SUBARU, TNG and HST) to study the variable star population of the remote Galactic globular cluster NGC 2419 and derive hints on a possible extragalactic origin for this cluster. We have discovered 40 new variables (34 RR Lyrae stars, 3 SX Phoenicis stars, 1 eclipsing binary system and 2 red irregular variables) doubling the number of known variable stars in NGC 2419.
\end{abstract}

Keywords. (Galaxy:) globular clusters: individual (NGC 2419);stars: variables: other

\section{Introduction}

NGC 2419, one of the most external globular clusters (GCs) in the Milky Way $\left(\mathrm{R}_{G C} \sim\right.$ $90 \mathrm{Kpc})$ is among the five brightest clusters in the Galaxy $\left(\mathrm{M}_{V}=-9.6 \mathrm{mag}\right)$. The cluster dynamical parameters (i.e core radius: $\mathrm{r}_{c} \sim 9 \mathrm{pc}$ ) are typical of an outer-halo cluster, however, NGC 2419, along with M 54 and $\omega$ Cen, has a high value of the half luminosity radius $\left(\mathrm{r}_{h}\right)$ and does not follow the correlation with luminosity holding for the other Galactic clusters (Mackey \& van den Berg, 2005). All these peculiarities and the similarity to $\omega$ Cen and M 54 suggest that NGC2419 might be the relic of a dwarf spheroidal galaxy. Substructures in the color magnitude diagrama (CMD) due to age/metallicity spreads of a possible composite population like that observed in $\omega$ Cen might not be observable in NGC 2419. Indeed, the cluster low metal abundance $([\mathrm{Fe} / \mathrm{H}]=-2.12$ dex compared to $[\mathrm{Fe} / \mathrm{H}]=-1.62$ of $\omega \mathrm{Cen})$ would make any metallicity spreads rather small and difficult to detect because NGC 2419 is significantly more distant than $\omega$ Cen. We are undertaking a comprehensive photometric study of NGC 2419 with the aim of testing the likeliwhood of an extragalactic origin, using: 1) the cluster variables and the RR Lyrae stars in particular, as population tracers, and 2) the CMDs at different radii from the cluster center to search for possible substructures around NGC 2419. Previous studies of the NGC 2419 variable stars date back to the work by Pinto \& Rosino (1997, PR) who collected photographic plates and detected 41 variables in NGC 2419, among which 25 fundamental mode pulsators (RRab), 6 first-overtones (RRc), 1 Population II Cepheid and 4 red irregular/semiregular variables. Based on the average period of the fundamental mode pulsators PR classified NGC 2419 as an Oosterhoff type II cluster (OoII, Oosterhoff 1939). 

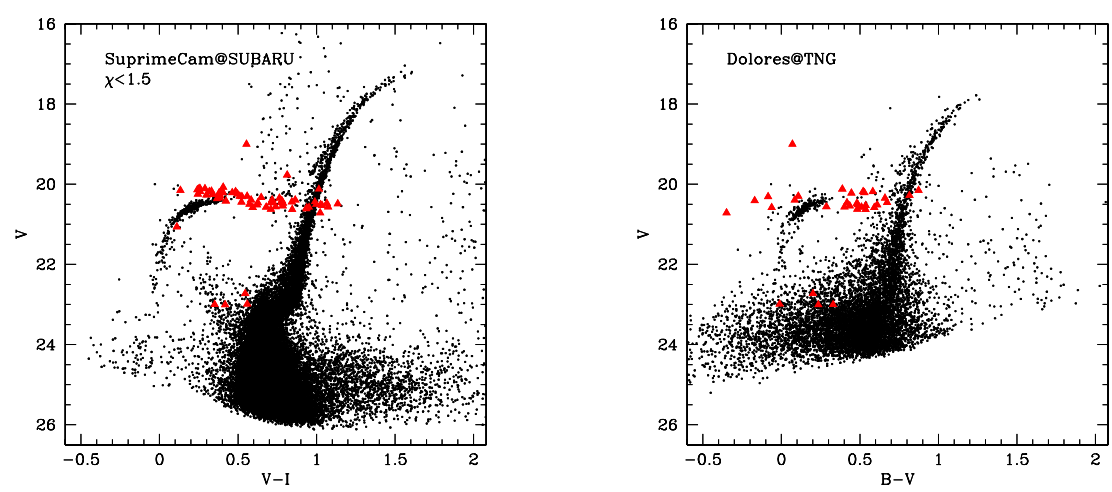

Figure 1. CMDs of NGC 2419, using SUBARU (left) and TNG (right) data. In both the panels variable stars are plotted with triangle and sampled at random phases.

\section{Discovery of new variables in NGC 2419}

We have performed a new study of the variables in NGC 2419 based on time series $V, I$ CCD photometry obtained with the SuprimeCam of the SUBARU $8 \mathrm{~m}$ telescope, $B, V$ images taken with DOLORES@TNG, and F555W(V), F814W(I) WFPC2@HST archival data. Homogeneous photometric reduction of the entire SUBARU+TNG+HST data set was performed with DAOPHOT-ALLSTAR-ALLFRAME (Stetson 1994, 1996). Variable stars have been identified with the Optimal Image Substraction package ISIS 2.1 (Alard 2000). We recovered all the previously known variables, and found 40 new variables in NGC 2419, they include: 32 RR Lyrae stars (among which 1 new double-mode pulsator and several first overtones), 3 SX Phoenicis stars, 1 eclipsing binary system, and 2 red irregular variables). Our study brings to 81 the total number of variable stars in NGC 2419. Figure 1 shows the $V, I$ and $B, V$ CMDs of NGC 2419 from the SUBARU and TNG datasets, respectively, with the variables marked by filled triangles. We show in Figure 2 the $V$ light curves of NGC 2419 newly discovered $a b$ - and $c$-type RR Lyrae stars. The next step will be the comparison of the pulsation properties of the full sample of NGC 2419 RR Lyrae stars with those of variables belonging to Galactic OoII clusters and to Local Group dwarf spheroidal galaxies.
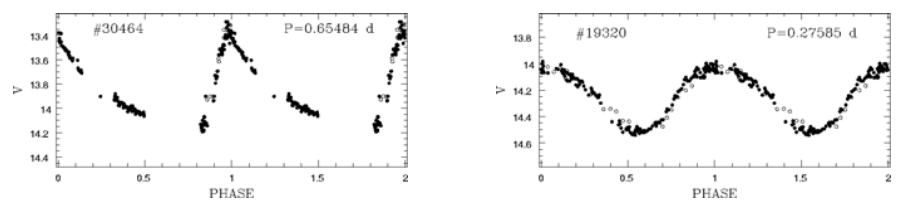

Figure 2. $V$ light curves of newly discovered $a b$ - (left) and $c$-type (right) RR Lyrae stars in NGC2419.

\section{References}

Alard, C. 2000, $A$ \& $A$ 144,363

Mackey \& van den Berg 2005, MNRAS 360, 631

Oosterhoff, P.Th. 1939, Observatory 62, 104

Pinto, G. \& Rosino, L. 1977, A \& A 28, 427

Stetson, P.B. 1994, PASP 106, 250

Stetson, P.B. 1996, User's Manual for DAOPHOT II 